\title{
A mathematical model for the dynamics of clustering
}

\author{
Dirk Aeyels, Filip De Smet \\ SYSTeMS Research Group, Dept. of Electrical Energy, Systems and Automation, \\ Ghent University, Technologiepark Zwijnaarde 914, 9052 Zwijnaarde, Belgium
}

\begin{abstract}
The formation of several clusters, arising from attracting forces between non-identical entities or agents, is a phenomenon observed in diverse fields. Think of people gathered through a mutual interest, swarm behavior of animals or clustering of oscillators in brain cells.

We introduce a dynamical model of mutually attracting agents for which we prove that the long term behavior consists of agents organized into several groups or clusters. We have completely characterized the cluster structure (i.e. the number of clusters and their composition) by means of a set of inequalities in the parameters of the model and have identified the intensity of the attraction as a key parameter governing the transition between different cluster structures.

The versatility of the model will be illustrated by discussing its relation to the Kuramoto model and by describing how it applies to a system of interconnected water basins.
\end{abstract}

Pacs numbers: 89.75.Fb, 05.65.+b, 05.45.Xt

Key words: multi-agent systems; self-organization; clustering; Kuramoto model

Published in: Physica D: Nonlinear Phenomena, Vol. 273, No 19, 2517-2530, October, 2008

DOI: $10.1016 /$ j.physd.2008.02.024

\section{Introduction and motivation}

The clustering phenomenon is observed in fields ranging from the exact sciences to social and life sciences; consider e.g. swarm behavior of animals or 
social insects [24], dynamics of opinion formation [12], or the clusters in the phase space for synchronized coupled oscillators [26] as a model for brain or heart cells. Swarming models mostly focus on the behavior and the cohesion of a single cluster $[4,19,11]$, and models for opinion formation often consider the coexistence of only two opposite opinions [27, 29, 3], although the emergence of multiple opinions has also been investigated [9]. For clustering in systems of coupled oscillators one distinguishes between phase clustering and frequency clustering. The first form (see e.g. [21, 10, 30]) is observed in a network of oscillators with identical natural frequencies. The clusters consist of oscillators with equal phases, while oscillators from different clusters have different phases. If the natural frequencies are not identical but their differences are sufficiently small then it may still be possible to distinguish different phase clusters [28]; this phenomenon is also related to multibranch entrainment [5]. Larger differences between the natural frequencies may induce oscillators having different long term average frequencies, resulting in frequency clustering [20]: each cluster is characterized by the long term average frequency of its members. This will be illustrated later on in this paper when we discuss the Kuramoto model. For more details on both phenomena and for examples of clustering in chaotic systems we refer to [18].

Due to the complexity and richness of some of these models, analytical results are often restricted to the existence and local stability properties of some of their solutions $[10,28,2]$, and exploration of the parameter space is usually done by simulations [11, 17]. The model we present in this paper (see also [1]) can be considered as a simplification of the Kuramoto model of coupled oscillators that retains its (frequency) clustering behavior. (A formal definition of clustering behavior is given later on.) Some other features typical for coupled oscillator models have disappeared; however, the potential for analytical results is greatly increased: for any choice of the parameters the emerging cluster structure can be characterized analytically. The model itself cannot be interpreted as a model for coupled oscillators (the interaction is not periodic), but it describes other applications, as we show in this paper.

In the next section we introduce the basic model. We also introduce an extended version of the model; see the forthcoming paper [7] which contains a detailed analysis and a generalization of the problem on compartmental systems, discussed in section 5 . In section 3 we describe the dynamical behavior of the solutions of the basic model. Section 4 contains a comparison with the Kuramoto model of coupled oscillators. We show that the behavior of our model is qualitatively similar to the behavior of the Kuramoto model. In section 5 we investigate a configuration of connected basins as a specific example of a compartmental system. Compartmental systems consist of interconnected compartments, each with a certain production or consumption rate. Multiple clusters may appear when the interconnections constrain the transport of a net production from one part of the network to another part 
with a net consumption. The substance involved may range from water to cars and from electrical energy to computer data. We will consider a system of interconnected water tanks, which is naturally modeled by the proposed model. Sections 6 to 9 contain details and proofs of the results.

\section{The dynamics}

In general interacting agents are bound to generate unpredictable unstructured behavior. We propose a mathematical model with a particular type of interaction such that through self-organization a structure emerges where sets of clustered agents find themselves in balance. The focus of this paper is on the dynamical behavior of this clustering phenomenon and on a characterization of the emerging cluster structure in terms of the parameters of the system.

We present a simple version admitting a succinct formulation of the conditions governing the cluster configuration at which the system settles. The differential equations for the model consisting of $N$ agents $(N>1)$ are

$$
\dot{x}_{i}(t)=b_{i}+\frac{1}{N} \sum_{j=1}^{N} f\left(x_{j}(t)-x_{i}(t)\right), \quad \forall t \in \mathbb{R}, \quad \forall i \in\{1, \ldots, N\},
$$

with $x_{i}(t), b_{i} \in \mathbb{R} ; b_{i}$ represents the autonomous component in each agent's behavior. The summation term represents the attraction exerted by the other agents on each agent, and depends on their mutual distances. The interaction function $f: \mathbb{R} \rightarrow \mathbb{R}$ is odd and non-decreasing. This implies a symmetric attraction between any two agents. We assume that the interaction intensifies with separation up to a certain saturation level:

$$
\exists d>0: f(x)=F, \quad \forall x \geq d
$$

In this paper we restrict our attention to this particular type of saturation; the general case will be discussed elsewhere [7]. A Lipschitz condition on $f$ is introduced to guarantee a unique solution to the differential equations with respect to a set of initial conditions.

The system equations are similar to those of the Kuramoto model, described by

$$
\dot{\theta}_{i}(t)=\omega_{i}+\frac{K}{N} \sum_{j=1}^{N} \sin \left(\theta_{j}(t)-\theta_{i}(t)\right), \quad \forall t \in \mathbb{R}, \quad \forall i \in\{1, \ldots, N\},
$$

where $\theta_{i}(t)$ denotes the phase of oscillator $i$ and $\omega_{i}$ its natural frequency. The behavior observed in simulations (see e.g. section 4, Figs. 1(a) and 1(c), or 
[6]) suggests that on a large time scale (i.e. averaged over a large time window) the interaction between the oscillators can be interpreted as a constant attraction, which explains the choice of the class of interaction functions $f$ in (1). Although with this simplification of the interaction function the model (1) cannot be interpreted as a model for coupled oscillators, a type of frequency clustering behavior remains true while a thorough analysis of the system becomes possible. The behavior of the Kuramoto model and its relation to the model (1) are discussed in section 4.

In a different physical context, the value $x_{i}(t)$ in (1) may also be interpreted as the concentration or quantity of a given substance at position $i$, with the interaction term $\frac{1}{N} f\left(x_{j}(t)-x_{i}(t)\right)$ equal to the flow from $j$ to $i$, limited to a maximum value of $\frac{F}{N}$. The flow may result from nonlinear diffusion (in the case of concentrations - with the flow being a nonlinear function of the concentration gradient, which is proportional to $x_{j}(t)-x_{i}(t)$ ), or from (hydrostatic) pressure differences as in the application in section 5 , but other mechanisms are possible. The value $b_{i}$ then represents the inflow at position $i$ from an external source, which is redistributed over the different compartments. More details are given in section 5 .

For convenience the results we present in this paper concern the model (1), but an extension to the following generalization is possible [7]:

$$
\dot{x}_{i}(t)=b_{i}+A_{i} \sum_{j=1}^{N} K_{i j} \gamma_{j} f\left(x_{j}(t)-x_{i}(t)\right), \quad \forall t \in \mathbb{R}, \quad \forall i \in\{1, \ldots, N\}
$$

The function $f$ has the same characteristics as before. The interpretation of $b_{i}$ remains unaltered. The parameters $A_{i}$ and $\gamma_{i}$ are all positive. The matrix $K$ is symmetric and irreducible with $K_{i j} \geq 0$. The elements of matrix $K$ represent levels of attraction between agent pairs; the extent to which each individual agent $j$ tends to attract other agents is denoted by $\gamma_{j}$. The parameter $A_{i}$ reflects the sensitivity of agent $i$ to interactions with other agents.

The interaction structure in (3) is not necessarily all-to-all and may be represented in graph theoretical terms: $K_{i j}$ corresponds to the weight of the edge of the associated graph with vertices $i$ and $j$ representing agents $i$ and $j$. If edges corresponding to $K_{i j}=0$ are omitted, then the irreducibility of the matrix $K$ is equivalent to the connectedness of this graph. The parameters $\gamma_{j}$ correspond to weights attached to the vertices. We refer to [7] for more details. 


\section{Analysis and results}

Assume that, for a particular solution of (1), the behavior of the agents can be characterized as follows by an ordered set of clusters $\left(G_{1}, \ldots, G_{M}\right)$ representing a partition of $\{1, \ldots, N\}$ :

(1) The distances between agents in the same cluster remain bounded (i.e. $\left|x_{i}(t)-x_{j}(t)\right|$ is bounded for all $i, j \in G_{k}$, for any $k \in\{1, \ldots, M\}$, for $t \geq 0)$.

(2) After some positive time $T$, the distances between agents in different clusters are at least $d$ and grow unbounded with time.

(3) The agents are ordered by their membership to a cluster: $k<l \Rightarrow x_{i}(t)<$ $x_{j}(t), \forall i \in G_{k}, \forall j \in G_{l}, \forall t \geq T$.

We will refer to this behavior as clustering behavior.

For any set $G_{0} \subset\{1, \ldots, N\}$, with the number of elements denoted by $\left|G_{0}\right|$, we introduce the notation $\langle b\rangle_{G_{0}}$ for the average value of $b_{i}$ over $G_{0}$ :

$$
\langle b\rangle_{G_{0}}=\frac{1}{\left|G_{0}\right|} \sum_{i \in G_{0}} b_{i} .
$$

In sections 6 and 7 we show that the following conditions are necessary and sufficient for clustering behavior of all solutions to the system (1), with the cluster structure $\left(G_{1}, \ldots, G_{M}\right)$ independent of the initial condition:

$$
\begin{gathered}
\langle b\rangle_{G_{k+1}}-\langle b\rangle_{G_{k}}>\frac{F}{N}\left(\left|G_{k+1}\right|+\left|G_{k}\right|\right), \quad \forall k \in\{1, \ldots, M-1\}, \quad \text { if } M>1, \\
\langle b\rangle_{G_{k, 2}}-\langle b\rangle_{G_{k, 1}} \leq \frac{F}{N}\left|G_{k}\right|, \quad \forall G_{k, 1}, G_{k, 2} \subsetneq G_{k}, \text { with } G_{k, 2}=G_{k} \backslash G_{k, 1}, \\
\quad \forall k \in\{1, \ldots, M\} .
\end{gathered}
$$

The conditions (4) state that the difference between the average natural velocities of subsequent clusters should be larger than the mutual attraction between the two clusters for these clusters to remain separated (condition (4a)). The difference in average natural velocities between any pair of subsets, partitioning a cluster, should not exceed the mutual attraction between these two subsets for a cluster to remain bounded (condition (4b)). Notice that these conditions only depend on the saturation value $F$, and not on the exact shape of the function $f$. Due to the characteristics of the interaction, each separate condition from (4) only contains the parameters of the clusters involved. However, the entire set of conditions (4) is necessary and sufficient for clustering behavior with respect to the entire cluster structure: the re- 
striction of the conditions to a limited number of clusters does not guarantee clustering behavior with respect to these clusters.

For a solution satisfying clustering behavior with clusters $G_{1}, \ldots, G_{M}$, it is easy to verify that the average velocity $\langle\dot{x}(t)\rangle_{G_{k}}$ over cluster $G_{k}$ will be constant after some time $T$ :

$$
\langle\dot{x}(t)\rangle_{G_{k}}=\langle b\rangle_{G_{k}}+\frac{F}{N}\left(\sum_{k^{\prime}>k}\left|G_{k^{\prime}}\right|-\sum_{k^{\prime}<k}\left|G_{k^{\prime}}\right|\right), \quad \forall t \geq T,
$$

which is explicitly shown in section 6 . Denote the right hand side by $v_{k}$. The velocity $v_{k}$ is equal to the sum of the average natural velocity of the agents in $G_{k}$, a positive term resulting from the attraction of the agents in the leading clusters, and a negative term resulting from the agents in the clusters behind. (Internal interactions - i.e. interactions between agents from the same cluster - cancel, since $f$ is odd.) From the boundedness of the distances between agents from the same cluster we can derive that clustering behavior of a solution $x$ with respect to $\left(G_{1}, \ldots, G_{M}\right)$ is equivalent with:

$$
\exists l>0:\left|x_{i}(t)-v_{k} t\right| \leq l, \quad \forall i \in G_{k}, \forall k \in\{1, \ldots, M\}, \forall t \geq 0 .
$$

Furthermore, from the analysis in section 9, where we investigate the internal behavior of the clusters, it will follow that for any $i \in G_{k}$, with $k \in\{1, \ldots, M\}, x_{i}(t)$ tends asymptotically to $v_{k} t$ plus a constant, or equivalently: $\lim _{t \rightarrow \infty}\left(x_{i}(t)-v_{k} t\right)$ exists. In other words: each agent asymptotically moves at a constant velocity. This behavior is similar to the behavior observed in simulations of the Kuramoto model, where the oscillators seem to exhibit a behavior governed by equations similar to (5).

In section 8 we show that for every given set of parameters $b_{i}$ and $F$ there exists a unique ordered partition $\left(G_{1}, \ldots, G_{M}\right)$ of clusters satisfying (4a) and (4b). This implies that for any choice of the parameters, the model (1) will exhibit clustering behavior with respect to some cluster structure. In general there exist $N-1$ bifurcation values for the intensity of attraction $F$, defining $N$ intervals for $F$; each interval corresponds to a particular cluster configuration, and transitions to new cluster configurations take place at these bifurcation points. In the generic case each transition (with decreasing $F$ ) corresponds to splitting one of the clusters into two.

As explained in section 9 it is useful to introduce a moving frame at the velocity of a cluster and consider the subsystem associated with the agents in this cluster. Under some mild assumptions this leads to a unique (up to a translation of all agents in the cluster over the same distance) asymptotically stable equilibrium point that will disappear with decreasing $F$ when the cluster splits into two. This motivates our use of the term 'bifurcation value' for those values of the coupling strength where a cluster splits. 
Given the model (1) and faced with the question of describing the cluster structure which eventually emerges, our analysis offers two options: either one checks the inequalities (4a) and (4b) or one simply runs a simulation of the model: our analysis guarantees convergence to a cluster structure, independent of the initial condition.

Remark 1 An important remark has to be made concerning the possible clusters that can arise from (1). Since there is all-to-all coupling it turns out that the order of the parameters $b_{i}$ determines the order of $x_{i}(t)$ in the long run, and therefore also the order of the asymptotic velocities $v_{k}$. This restricts the possible cluster configurations: a cluster containing agents $i_{1}$ and $i_{2}$ will then also contain agent $i_{3}$ whenever $b_{i_{1}} \leq b_{i_{3}} \leq b_{i_{2}}$. As a consequence in (4b) $G_{k, 1}$ (resp. $G_{k, 2}$ ) can be restricted to contain only the agents of $G_{k}$ with the $\left|G_{k, 1}\right|$ lowest (resp. $\left|G_{k, 2}\right|$ highest) $b_{i}$-values. In the more general case described by (3) this remark is not necessarily true.

In a forthcoming paper [7] we will extend the results in this paper to the more general system (3).

We now discuss the relation to the Kuramoto model and we describe an application on compartmental systems. The final sections contain the mathematical details of the results stated above.

\section{The Kuramoto model}

The Kuramoto model [14] is a mathematical model describing systems of coupled oscillators; one is referred to [26] for its relation to flashing fireflies, pacemaker cells, Josephson junctions. The differential equations corresponding to a system of $N$ oscillators with phases $\theta_{i}$ are given by (2). The natural frequencies $\omega_{i}$ are drawn randomly from some distribution $g$. In [14] it is shown that in the limit $N \rightarrow \infty$ and with $g$ unimodal and even about a value $\Omega$, there is a critical value for the coupling strength $K$ above which a solution exists exhibiting partial synchronization: this solution is characterized by a group of oscillators (with $\omega_{i}$-values close to $\Omega$ ) moving at the same (constant) frequency $\Omega$, while the remaining oscillators are moving with different (long term average) frequencies. Details can be found in [25].

For finite $N$, simulations indicate the following. For a fixed $K>0$ the oscillator population can be partitioned into different subsets of which the members have bounded phase differences: the system exhibits partial entrainment. It immediately follows that oscillators belonging to the same entrained subset have the same long term average frequency. The partition and the associated average frequencies are independent of the initial condition for most (but 
not all — see e.g. [17]) choices of the natural frequencies and the coupling strength. For small values of $K$ and all $\omega_{i}$ different, the entrained subsets are singletons and the associated average frequencies are close to the $\omega_{i}$-values. For increasing coupling strength $K$ the characteristic frequencies move towards each other and when a critical value for $K$ is passed and two characteristic frequencies coincide, the union of the corresponding entrained subsets also becomes entrained. In the most common scenario this phenomenon is selfrepeating and after $N-1$ transitions there is full entrainment, i.e. all phase differences are bounded. For more information on the stability properties of this latter solution, see [2].

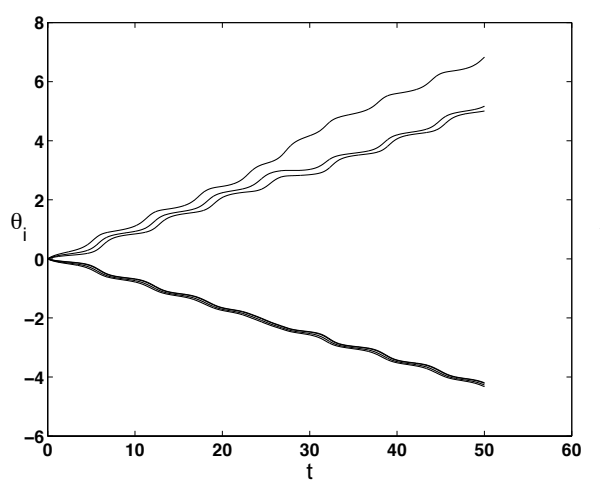

(a)

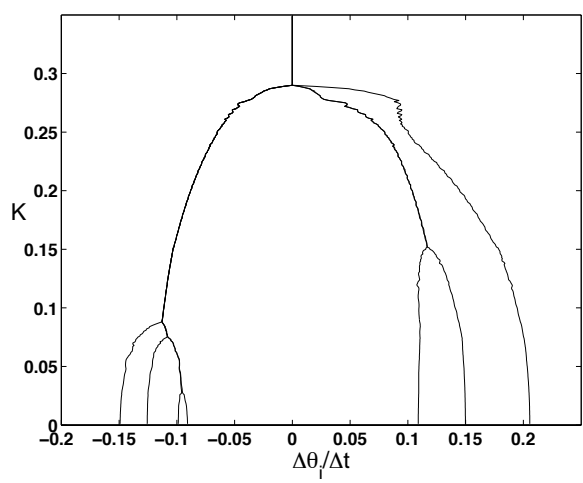

(c)

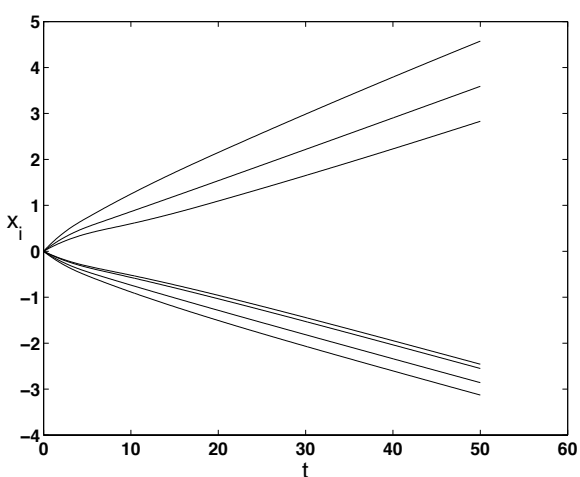

(b)

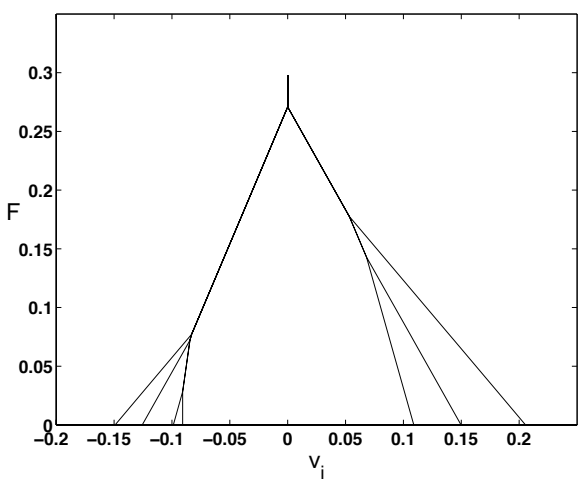

(d)

Fig. 1. Comparison of the Kuramoto model (left column) with the model (1) (right column): The first row shows the time evolution of the oscillators/agents. The second row shows the evolution of the long term average frequencies/velocities for varying coupling strength. The parameters are: $b=\omega=(-0.149,-0.126,-0.099,-0.091$, $0.109,0.150,0.206$ ), $K=0.2$ (Fig. (a)) and $F=0.15$ (Fig. (b)).

This behavior is very similar to that of the model (1), where the clusters are independent of the initial condition and the transitions between the different clusters for varying $F$ are similar. For comparison, Fig. 1 shows the time evolution for a particular configuration for the Kuramoto model (Fig. 1(a)) and the analogue for the model (1) (Fig. 1(b)), as well as the evolution of the characteristic frequencies/velocities for both models in terms of the coupling 
strength (Fig. 1(c) and 1(d)). The qualitative correspondence between both models is remarkable.

Besides clustering behavior, the Kuramoto model also exhibits some phenomena such as frequency locking [17] or induction of clusters by resonances [8], which are not present in the model (1). However, this richer behavior of the Kuramoto model has its price: in spite of the simplicity of its formulation, the model is complicated and hard to analyze. The model (1) allows us to focus on clustering behavior, while admitting a full analysis.

\section{Compartmental systems: interconnected water basins}

To illustrate the relation of our clustering model with compartmental systems we will focus on a system of interconnected water basins.

We consider $N$ identical basins connected by horizontal identical pipes, each basin connected to all other basins and furthermore subject to either a constant external inflow or outflow of water. For laminar flow through a pipe connecting two basins, the fluid velocity is proportional to the pressure difference (Hagen-Poiseuille law). For larger velocities the flow becomes turbulent and the relation between velocity and pressure difference is described by the Darcy-Weisbach equation [23]:

$$
\Delta p=\lambda \frac{L}{D} \frac{\rho v^{2}}{2}
$$

with $\Delta p$ the pressure difference, $L$ and $D$ length and diameter of the pipe, $\rho$ the fluid density, $v$ the mean fluid velocity (i.e. the ratio of the volume flow rate and the cross-section area), and $\lambda$ the friction factor. Although the friction factor depends on the Reynolds number (which is proportional to the fluid velocity) its variation is small for large values of the Reynolds number. We will approximate the resulting relation $(v(\Delta p) \sim \pm \sqrt{|\Delta p|})$ by an odd saturating function, keeping in mind that the flow rate cannot grow unbounded (because of the finite basin heights and finite resulting pressures). In other words, we assume that the pipes have a maximal throughput, which is independent of the direction of the flow, and denoted by $T$. Representing the water height of basin $i$ by $x_{i}$, the pressure difference between basins $i$ and $j$ will be proportional to $x_{j}-x_{i}$, and thus the volume flow rate through the connecting pipe can be represented by $\frac{S}{N} f\left(x_{j}-x_{i}\right)$, with $S$ denoting the surface area of the basins which we assume to be water level independent — and the saturation value $F$ of $f$ equal to $\frac{N T}{S}$. 
Denoting the inflow for basin $i$ by $Q_{i}$, one derives

$$
\dot{x}_{i}(t)=\frac{Q_{i}}{S}+\frac{1}{N} \sum_{j=1}^{N} f\left(x_{j}(t)-x_{i}(t)\right), \quad \forall t \in \mathbb{R}, \quad \forall i \in\{1, \ldots, N\},
$$

which is the model (1) with $b_{i}=\frac{Q_{i}}{S}$ for all $i$. With the extended model (3) more general network structures and non-identical basins can be taken into account (see [7]).

The dynamics will exhibit clustering behavior as predicted by our analysis, in the sense that the water level heights of some of the basins will (asymptotically) increase/decrease with the same velocity. The velocity associated with cluster $G_{k}$ is denoted by $v_{k}$.

It is clear that the model is valid as long as the basins do not overflow and all pipes remain below the water level of the basins they connect. We assume that the basins and initial water level heights are such that these conditions are satisfied during the transient behavior. As explained in the next paragraph, this implies that we can derive the behavior of the physical system (i.e. overflowing basins, or basins running empty) from the cluster structure of the mathematical model and the corresponding long term velocities $v_{k}$.

The problem we are interested in is to check whether a network of basins is prone to flooding, i.e. one requires $v_{k} \leq 0$ for all clusters $G_{k}$. Assume from now on that the total external inflow equals the total external outflow. When for this case the dynamical behavior of the model reveals the existence of more than one cluster, there would be at least one cluster $G_{k}$ corresponding to flooding, with a positive value for $v_{k}$. Basins will overflow and after some time the model will no longer represent the physical situation. However, the model (1) continues to be useful for investigating compartmental systems and revealing problematic situations with respect to flooding. Indeed, a simulation of the mathematical model, although only valid in a finite time interval, may reveal in its long term behavior the existence of multiple clusters: the overflowing basins in the real world system correspond to the clusters with a positive $v_{k}$.

For a solution with two clusters $G_{1}$ and $G_{2}$ (with $v_{1}$ negative and $v_{2}$ positive), the interpretation of the corresponding inequality (4a) is that the production in the basins belonging to $G_{2}$ cannot be transported to the basins belonging to $G_{1}$ by their interconnections.

Under the assumption of balanced in- and outflow, a solution with a single cluster $G_{1}=\{1, \ldots, N\}$, has a corresponding velocity equal to $v_{1}=0$. This is a case where no flooding will occur, no basins will run empty, and the model will remain valid for all positive time, with each $x_{i}$ approaching a constant value. 
As an illustration, consider a configuration of $N=5$ basins with surface area $S=1$, implying that $b_{i}=Q_{i}$. (For simplicity we will omit units.) The vector $b$ containing the $b_{i}$-values is given by

$$
b=\left[\begin{array}{lllll}
-5 & 2 & 1 & -2 & 4
\end{array}\right]^{T} .
$$

In order to find the lowest possible throughput $T=\frac{S}{N} F=\frac{F}{5}$ for which flooding is avoided, we can consider the inequalities (4b), taking into account remark 1:

$$
\begin{aligned}
\frac{5}{4}-(-5) & \leq F \\
\frac{7}{3}-\left(-\frac{7}{2}\right) & \leq F \\
3-(-2) & \leq F \\
4-(-1) & \leq F,
\end{aligned}
$$

resulting in $F \geq \frac{25}{4}$, or $T \geq \frac{5}{4}$. This can be checked in the bifurcation diagram in Fig. 2.

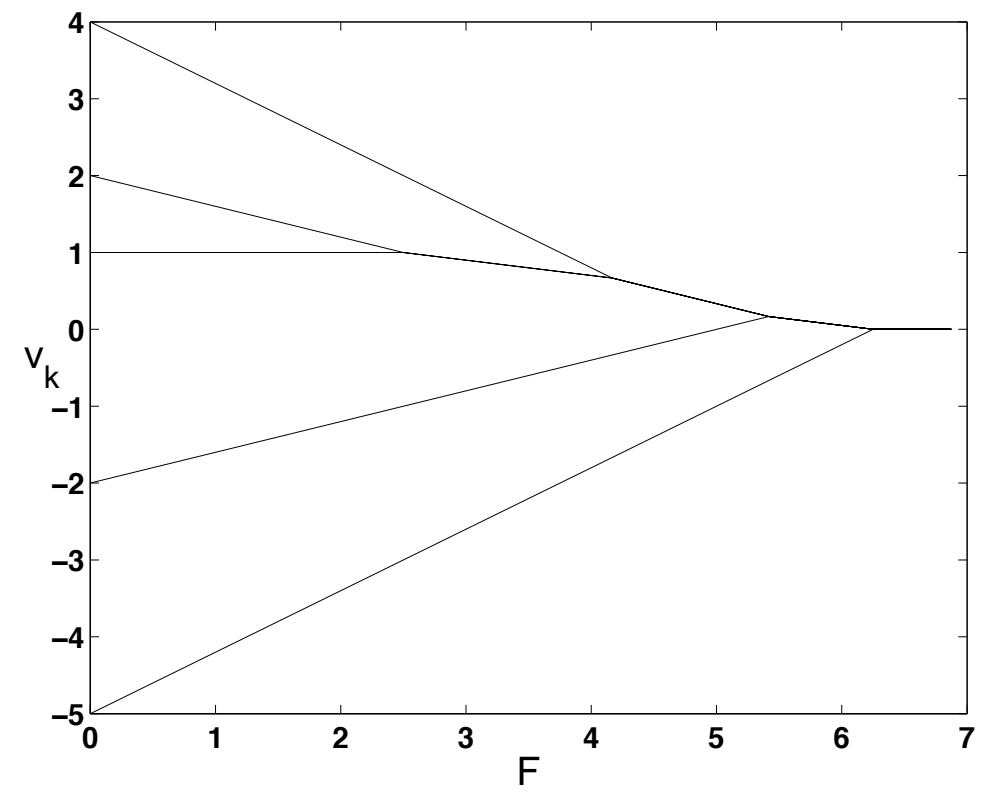

Fig. 2. Asymptotic velocities in terms of the coupling strength $F$.

For smaller values of $T$ and $F$ some of the basins will overflow. Given a particular value of $F$ smaller than $\frac{25}{4}$, the overflowing basins can be identified from Fig. 2 (since the intersection with the vertical axis yields the corresponding value of $b_{i}$ ). It may be intuitively obvious that these basins will correspond to the highest $b_{i}$-values, as can also be inferred from remark 1 . For general network structures described by (3) this may no longer be true (see [7]). 


\section{The conditions (4) are necessary for the existence of clustering behavior}

We show that the existence of a solution of the system (1) exhibiting clustering behavior with clusters $G_{1}, \ldots, G_{M}$ implies the conditions (4).

Let $G_{0} \subset\{1, \ldots, N\}$ and consider $\langle\dot{x}(t)\rangle_{G_{0}}$; since $f$ is odd, all internal interactions (i.e. interactions between agents in $G_{0}$ ) will cancel in the expression for $\langle\dot{x}(t)\rangle_{G_{0}}$. Assume that, at some time instance $t_{0}$, the agents in $G_{0}$ are separated by at least a distance $d$ from all other agents (i.e. $\left|x_{i}\left(t_{0}\right)-x_{j}\left(t_{0}\right)\right| \geq d$ whenever $i \in G_{0}$ and $\left.j \notin G_{0}\right)$. Then the interactions of agents in $G_{0}$ with agents not in $G_{0}$ are all saturated. Assume furthermore that at $t_{0}$ each agent not belonging to $G_{0}$ has a $x\left(t_{0}\right)$-value either smaller or larger than all $x\left(t_{0}\right)$-values of agents in $G_{0}$. Denote by $G_{-}$(resp. $\left.G_{+}\right)$the set of agents with $x\left(t_{0}\right)$-values smaller (resp. larger) than the $x\left(t_{0}\right)$-values of the agents in $G_{0}$. Then

$$
\begin{aligned}
\left\langle\dot{x}\left(t_{0}\right)\right\rangle_{G_{0}} & =\langle b\rangle_{G_{0}}+\frac{1}{\left|G_{0}\right| N} \sum_{i \in G_{0}} \sum_{j=1}^{N} f\left(x_{j}\left(t_{0}\right)-x_{i}\left(t_{0}\right)\right) \\
& =\langle b\rangle_{G_{0}}+\frac{1}{\left|G_{0}\right| N} \sum_{i \in G_{0}}\left(\sum_{j \in G_{-}} f\left(x_{j}\left(t_{0}\right)-x_{i}\left(t_{0}\right)\right)+\sum_{j \in G_{+}} f\left(x_{j}\left(t_{0}\right)-x_{i}\left(t_{0}\right)\right)\right) \\
& =\langle b\rangle_{G_{0}}-\frac{1}{\left|G_{0}\right| N}\left|G_{0}\right|\left|G_{-}\right| F+\frac{1}{\left|G_{0}\right| N}\left|G_{0}\right|\left|G_{+}\right| F
\end{aligned}
$$

and thus

$$
\left\langle\dot{x}\left(t_{0}\right)\right\rangle_{G_{0}}=\langle b\rangle_{G_{0}}+\frac{F}{N}\left(\left|G_{+}\right|-\left|G_{-}\right|\right) .
$$

Assume now that the solution $x$ exhibits clustering behavior with cluster structure $\left(G_{1}, \ldots, G_{M}\right)$. Then $d$-separation of agents belonging to different clusters implies that we can apply the formula (6), for $t \geq T$, to each of the clusters $G_{k}(k \in\{1, \ldots, M\})$, and thus $\langle x\rangle_{G_{k}}$ will be an affine function of time. Furthermore, the ordering of agents belonging to different clusters and distances growing unbounded with positive time for agents in different clusters imply that for successive clusters $G_{k}$ and $G_{k+1},\langle\dot{x}\rangle_{G_{k}}<\langle\dot{x}\rangle_{G_{k+1}}$. But

$$
\begin{aligned}
\langle\dot{x}(t)\rangle_{G_{k+1}}-\langle\dot{x}(t)\rangle_{G_{k}}=\langle b\rangle_{G_{k+1}}+\frac{F}{N}\left(\sum_{k^{\prime}>k+1}\left|G_{k^{\prime}}\right|-\sum_{k^{\prime}<k+1}\left|G_{k^{\prime}}\right|\right) \\
\quad-\langle b\rangle_{G_{k}}-\frac{F}{N}\left(\sum_{k^{\prime}>k}\left|G_{k^{\prime}}\right|-\sum_{k^{\prime}<k}\left|G_{k^{\prime}}\right|\right) \\
=\langle b\rangle_{G_{k+1}}-\langle b\rangle_{G_{k}}-\frac{F}{N}\left(\left|G_{k+1}\right|+\left|G_{k}\right|\right),
\end{aligned}
$$


for $t \geq T$, by application of (6). Therefore $\langle\dot{x}\rangle_{G_{k}}<\langle\dot{x}\rangle_{G_{k+1}}$ can be written as (4a).

Under the assumption of clustering behavior the distances within one cluster are bounded, which implies that for any nonempty proper subset $G_{k, 1}$ of $G_{k}$, $\left|\langle x(t)\rangle_{G_{k, 2}}-\langle x(t)\rangle_{G_{k, 1}}\right|$ (with $G_{k, 2}=G_{k} \backslash G_{k, 1}$ ) is bounded for $t \geq 0$. Furthermore, because of the $d$-separation (after some time $T$ ) with agents not belonging to $G_{k}$ and because of the properties of $f$ there is a lower bound to $\langle\dot{x}(t)\rangle_{G_{k, 2}}-\langle\dot{x}(t)\rangle_{G_{k, 1}}$ corresponding to the situation where all agents of $G_{k, 2}$ have $x$-values that are at least $d$ larger than the $x$-values of the agents in $G_{k, 1}$. Again invoking (6) we calculate this lower bound as

$$
\langle b\rangle_{G_{k, 2}}-\langle b\rangle_{G_{k, 1}}-\frac{F}{N}\left|G_{k}\right|
$$

This value cannot be positive, otherwise $\langle x(t)\rangle_{G_{k, 2}}-\langle x(t)\rangle_{G_{k, 1}}$ would grow unbounded, contradicting the assumption that $G_{k}$ is bounded. The assumption of clustering behavior therefore implies the condition (4b). We conclude that the inequalities (4a) and (4b) are necessary conditions for the existence of a solution of (1) satisfying clustering behavior with respect to $\left(G_{1}, \ldots, G_{M}\right)$. They also turn out to be sufficient, as is shown next.

\section{The conditions (4) are sufficient for the existence of clustering behavior}

\subsection{A particular initial condition}

We show that, under the conditions (4) for some ordered set partition $G=$ $\left(G_{1}, \ldots, G_{M}\right)$, there is an initial condition such that the corresponding solution of the system (1) exhibits clustering behavior with respect to $G$.

When agents from different sets $G_{k}$ are separated over a distance of at least $d$, their interaction is saturated; if the order of the agents is also determined by the order of the sets $G_{k}$, then the total interaction of agents outside $G_{k}$ with an agent from $G_{k}$ equals $\frac{F}{N}\left(\sum_{k^{\prime}>k}\left|G_{k^{\prime}}\right|-\sum_{k^{\prime}<k}\left|G_{k^{\prime}}\right|\right)$. To investigate the behavior of (1) under the assumption of saturated attraction between agents belonging to different sets $G_{k}$, consider the following auxiliary system:

$$
\begin{aligned}
\dot{x}_{i}^{*}(t)=b_{i}+\frac{F}{N}\left(\sum_{k^{\prime}>k}\left|G_{k^{\prime}}\right|-\sum_{k^{\prime}<k}\left|G_{k^{\prime}}\right|\right)+\frac{1}{N} \sum_{j \in G_{k}} f\left(x_{j}^{*}(t)-x_{i}^{*}(t)\right), \\
\forall t \geq 0, \forall i \in G_{k}, \\
\forall k \in\{1, \ldots, M\} .
\end{aligned}
$$


Let $x$, resp. $x^{*}$, be the solution of the system (1), resp. (7), corresponding to the initial condition $x(0)=x^{*}(0)$, with $x_{i}(0)=x_{j}(0)$ whenever $i$ and $j$ belong to the same set $G_{k}$. Further specification of $x_{i}(0)$ and $x_{j}(0)$ with $i$ and $j$ belonging to different sets $G_{k}$ will be imposed according to (12).

We first show that (4b) implies that $\left|x_{i}^{*}(t)-x_{j}^{*}(t)\right|\left(i, j \in G_{k}, k \in\{1, \ldots, M\}\right)$ is bounded. Consider the region $R \subset \mathbb{R}^{N}$ defined by

$$
\begin{aligned}
y \in R \Leftrightarrow\langle y\rangle_{G_{k, 2}}-\langle y\rangle_{G_{k, 1}} \leq\left|G_{k}\right| \frac{d}{2} & \\
& \forall G_{k, 1}, G_{k, 2} \subsetneq G_{k} \text { with } G_{k, 2}=G_{k} \backslash G_{k, 1}, \\
& \forall k \in\{1, \ldots, M\} .
\end{aligned}
$$

Clearly $x(0)=x^{*}(0) \in R$. Assume that, for some $\tilde{t} \geq 0, x^{*}(\tilde{t}) \in R$ is at the boundary of $R$ : there exist $\tilde{k} \in\{1, \ldots, M\}, G_{\tilde{k}, 1}=\tilde{G}_{1}, G_{\tilde{k}, 2}=\tilde{G}_{2}$ such that

$$
\left\langle x^{*}(\tilde{t})\right\rangle_{\tilde{G}_{2}}-\left\langle x^{*}(\tilde{t})\right\rangle_{\tilde{G}_{1}}=\left|G_{\tilde{k}}\right| \frac{d}{2} .
$$

Application of the inequalities in (8) to $x^{*}(\tilde{t})$ for $G_{\tilde{k}, 1}=\tilde{G}_{1} \backslash\{i\}$ resp. $G_{\tilde{k}, 1}=$ $\tilde{G}_{1} \cup\{j\}\left(i \in \tilde{G}_{1}, j \in \tilde{G}_{2}\right)$ leads to

$$
\begin{aligned}
\left\langle x^{*}(\tilde{t})\right\rangle_{\tilde{G}_{2} \cup\{i\}}-\left\langle x^{*}(\tilde{t})\right\rangle_{\tilde{G}_{1} \backslash\{i\}} & \leq\left|G_{\tilde{k}}\right| \frac{d}{2}, \\
\left\langle x^{*}(\tilde{t})\right\rangle_{\tilde{G}_{2} \backslash\{j\}}-\left\langle x^{*}(\tilde{t})\right\rangle_{\tilde{G}_{1} \cup\{j\}} & \leq\left|G_{\tilde{k}}\right| \frac{d}{2} .
\end{aligned}
$$

Multiplication of (10a) with $\left(\left|\tilde{G}_{2}\right|+1\right)\left(\left|\tilde{G}_{1}\right|-1\right)$, (10b) with $\left(\left|\tilde{G}_{2}\right|-1\right)\left(\left|\tilde{G}_{1}\right|+1\right)$, (9) with $-2\left|\tilde{G}_{2}\right|\left|\tilde{G}_{1}\right|$, and addition eventually results in

$$
x_{i}^{*}(\tilde{t}) \leq x_{j}^{*}(\tilde{t})-d, \quad \forall i \in \tilde{G}_{1}, \forall j \in \tilde{G}_{2} .
$$

It follows that the interactions between agents from $\tilde{G}_{1}$ and agents from $\tilde{G}_{2}$ are saturated and

$$
\begin{aligned}
\left\langle\dot{x}^{*}(\tilde{t})\right\rangle_{\tilde{G}_{2}}-\left\langle\dot{x}^{*}(\tilde{t})\right\rangle_{\tilde{G}_{1}} & =\langle b\rangle_{\tilde{G}_{2}}-\langle b\rangle_{\tilde{G}_{1}}-\frac{F}{N}\left|G_{\tilde{k}}\right|, & & \text { by }(7), \\
& \leq 0, & & \text { by }(4 \mathrm{~b}),
\end{aligned}
$$

and thus $\left\langle x^{*}\right\rangle_{\tilde{G}_{2}}-\left\langle x^{*}\right\rangle_{\tilde{G}_{1}}$ cannot increase at time $\tilde{t}$. This implies that if $x^{*}\left(t_{0}\right) \in$ $R$ for some $t_{0} \geq 0$, then $x^{*}(t)$ will remain in $R$ for $t \geq t_{0}: R$ is a trapping region for system (7).

Since $x^{*}(0) \in R$, it then follows that $x^{*}(t) \in R, \forall t \geq 0$. From (8) with $G_{k, 1}=\{i\}$ resp. $G_{k, 2}=\{i\}$ it follows that

$$
\left|x_{i}^{*}(t)-\left\langle x^{*}(t)\right\rangle_{G_{k} \backslash\{i\}}\right| \leq\left|G_{k}\right| \frac{d}{2}, \quad \forall t \geq 0, \forall i \in G_{k}, \forall k \in\{1, \ldots, M\},
$$


which is equivalent to

$$
\left|x_{i}^{*}(t)-\left\langle x^{*}(t)\right\rangle_{G_{k}}\right| \leq\left(\left|G_{k}\right|-1\right) \frac{d}{2}, \quad \forall t \geq 0, \forall i \in G_{k}, \forall k \in\{1, \ldots, M\},
$$

and thus, setting $C \triangleq(N-1) d$,

$$
\left|x_{i}^{*}(t)-x_{j}^{*}(t)\right| \leq C, \quad \forall i, j \in G_{k}, \forall k \in\{1, \ldots, M\},
$$

for all $t \geq 0$, by the triangle inequality.

Consider now the system (1). Pick $\epsilon>0$ and further specify the initial condition $x(0)$ by imposing that

$$
\langle x(t)\rangle_{G_{k}}+C \leq\langle x(t)\rangle_{G_{k+1}}-C-d-\epsilon, \quad \forall k \in\{1, \ldots, M-1\},
$$

for $t=0$. This implies that, at $t=0$,

$$
x_{i}(t) \leq x_{j}(t)-d-\epsilon, \quad \forall i \in G_{k}, j \in G_{k+1}, \forall k \in\{1, \ldots, M-1\},
$$

since $x(0)=x^{*}(0)$ and because of (11). From (1) it follows that all $\left|\dot{x}_{i}\right|$ are bounded (for instance by the value $\max _{i}\left|b_{i}\right|+F$ ) and thus there exists a $\Delta t>0$ such that for $t \in[0, \Delta t]$

$$
x_{i}(t) \leq x_{j}(t)-d, \quad \forall i \in G_{k}, j \in G_{k+1}, \forall k \in\{1, \ldots, M-1\} .
$$

We have shown that in the time interval $[0, \Delta t]$, the agents follow the ordering of the sets $G_{k}$, with agents from different sets $G_{k}$ separated over a distance of at least $d$ (as required by the definition of clustering behavior).

Whenever (13b) holds, the differential equations (1) and (7) are equivalent. Consequently, for all $t \in[0, \Delta t], x(t)$ and $x^{*}(t)$ are equal, implying

$$
\left|x_{i}(t)-x_{j}(t)\right| \leq C, \quad \forall i, j \in G_{k}, \forall k \in\{1, \ldots, M\} .
$$

Notice that because of (13b), expression (6) can be applied to each set $G_{k}$ and as a consequence the condition (4a) implies that $\langle\dot{x}(t)\rangle_{G_{k+1}}-\langle\dot{x}(t)\rangle_{G_{k}}>0$ for $t \in[0, \Delta t]$, and thus (12) will also hold for $t \in[0, \Delta t]$. For all $t \in[0, \Delta t],(12)$ and (14) hold and thus also (13a). Since we can repeat this argument starting from time $n \Delta t(n=1,2, \ldots)$, it follows that (13b) and (14) hold for all $t \in \mathbb{R}^{+}$; this accounts for the boundedness and the ordering of the agents according to the sets $G_{k}$. Furthermore, from the application of (6) to the sets $G_{k}$ together with (4a) and (14) for all $t \geq 0$, it follows that the distances between agents of different $G_{k}$ will be unbounded in time. This leads us to conclude that the solution corresponding to the specified initial condition will indeed exhibit clustering behavior with $T=0$ and with the clusters corresponding to the sets $G_{k}$. 


\subsection{Independence of the initial condition}

Let $x$ denote the particular solution from the previous section and let $\hat{x}$ denote any other solution of (1). The square of the distance in the state space between $x(t)$ and $\hat{x}(t)$ is

$$
V(t) \triangleq \sum_{i=1}^{N}\left(x_{i}(t)-\hat{x}_{i}(t)\right)^{2}
$$

Taking the time derivative of the function $V$ gives

$$
\begin{aligned}
\dot{V}(t)= & 2 \sum_{i=1}^{N}\left(x_{i}(t)-\hat{x}_{i}(t)\right)\left(\frac{1}{N} \sum_{j=1}^{N} f\left(x_{j}(t)-x_{i}(t)\right)-\frac{1}{N} \sum_{j=1}^{N} f\left(\hat{x}_{j}(t)-\hat{x}_{i}(t)\right)\right) \\
= & 2 \frac{1}{N} \sum_{i=1}^{N} \sum_{j=1}^{N}\left(x_{i}(t)-\hat{x}_{i}(t)\right)\left(f\left(x_{j}(t)-x_{i}(t)\right)-f\left(\hat{x}_{j}(t)-\hat{x}_{i}(t)\right)\right) \\
= & \frac{1}{N} \sum_{i=1}^{N} \sum_{j=1}^{N}\left(\left(x_{i}(t)-\hat{x}_{i}(t)\right)\left(f\left(x_{j}(t)-x_{i}(t)\right)-f\left(\hat{x}_{j}(t)-\hat{x}_{i}(t)\right)\right)\right. \\
& \left.\quad+\left(x_{j}(t)-\hat{x}_{j}(t)\right)\left(f\left(x_{i}(t)-x_{j}(t)\right)-f\left(\hat{x}_{i}(t)-\hat{x}_{j}(t)\right)\right)\right) \\
= & -\frac{1}{N} \sum_{i=1}^{N} \sum_{j=1}^{N}\left(\left(x_{j}(t)-x_{i}(t)\right)-\left(\hat{x}_{j}(t)-\hat{x}_{i}(t)\right)\right) \\
\leq & 0, \quad \times\left(f\left(x_{j}(t)-x_{i}(t)\right)-f\left(\hat{x}_{j}(t)-\hat{x}_{i}(t)\right)\right),
\end{aligned}
$$

because both factors always have the same sign, since $f$ is non-decreasing. Therefore $V(t) \leq V(0)$, and thus $\left|x_{i}(t)-\hat{x}_{i}(t)\right| \leq \sqrt{V(0)}, \forall t \geq 0, \forall i \in$ $\{1, \ldots, N\}$, which, together with the clustering behavior for $x$, implies that $\hat{x}$ also exhibits clustering behavior. We conclude that conditions (4) characterize clustering behavior of all solutions, independent of the initial conditions.

Remark 2 As $V(t)$ equals the square of the distance between $x(t)$ and $\hat{x}(t)$, the implication that $\dot{V} \leq 0$ leads to a unique clustering behavior for all initial conditions is strongly related to the notions of 'convergent systems' [22] and 'contracting systems' [16]. However, the system (1) exhibits a weaker property than uniform contraction (which is imposed in [22, 16] and related literature), and consequently the function $V$ does not necessarily approach zero. 


\section{Existence and uniqueness of the cluster partition with respect to $b$ and $F$}

We will prove that, given $b$ and $F \geq 0$, there corresponds a unique ordered partition $G=\left(G_{1}, \ldots, G_{M}\right)$ for which (4) holds. For large values of $F$ (4) is satisfied for $G=(\{1, \ldots, N\})$ (corresponding to only one cluster). As will be shown in lemma 4 , whenever for a given cluster structure - satisfying (4) one of the conditions (4b) ceases to hold with decreasing $F$ (obviously (4a) will remain satisfied), there is always another unique ordered set partition for which (4) will hold again. This will be a key element in the proof of the following theorem.

Theorem 3 For each $b \in \mathbb{R}^{N}$ and each $F \geq 0$, there exists a unique ordered set partition $G$ of $\{1, \ldots, N\}$, such that (4) holds.

First we consider the following lemma, which is proven in section A of the appendix.

Lemma 4 For any ordered set partition $G=\left(G_{1}, \ldots, G_{M}\right)$ satisfying

$$
\begin{aligned}
& \langle b\rangle_{G_{k+1}}-\langle b\rangle_{G_{k}} \geq \frac{F}{N}\left(\left|G_{k+1}\right|+\left|G_{k}\right|\right), \quad \forall k \in\{1, \ldots, M-1\}, \\
& \langle b\rangle_{G_{k, 2}}-\langle b\rangle_{G_{k, 1}} \leq \frac{F}{N}\left|G_{k}\right|, \quad \forall G_{k, 1}, G_{k, 2} \subsetneq G_{k}, \text { with } G_{k, 2}=G_{k} \backslash G_{k, 1}, \\
& \quad \forall k \in\{1, \ldots, M\},
\end{aligned}
$$

it is true that if for some $k^{\prime} \in\{1, \ldots, M\}$ and some $G_{k^{\prime}, 1}, G_{k^{\prime}, 2} \subsetneq G_{k^{\prime}}$, with $G_{k^{\prime}, 2}=G_{k^{\prime}} \backslash G_{k^{\prime}, 1}$

$$
\langle b\rangle_{G_{k^{\prime}, 2}}-\langle b\rangle_{G_{k^{\prime}, 1}}=\frac{F}{N}\left|G_{k^{\prime}}\right|
$$

then the cluster structure $G^{\prime} \triangleq\left(G_{1}^{\prime}, \ldots, G_{k^{\prime}-1}^{\prime}, G_{k^{\prime}}^{\prime}, G_{k^{\prime}+1}^{\prime}, G_{k^{\prime}+2}^{\prime}, \ldots, G_{M+1}^{\prime}\right) \triangleq$ $\left(G_{1}, \ldots, G_{k^{\prime}-1}, G_{k^{\prime}, 1}, G_{k^{\prime}, 2}, G_{k^{\prime}+1}, \ldots, G_{M}\right)$ satisfies

$$
\begin{aligned}
& \langle b\rangle_{G_{k+1}^{\prime}}-\langle b\rangle_{G_{k}^{\prime}} \geq\left(\left|G_{k+1}^{\prime}\right|+\left|G_{k}^{\prime}\right|\right) \frac{F}{N}, \quad \forall k \in\{1, \ldots, M\} \backslash\left\{k^{\prime}\right\}, \\
& \langle b\rangle_{G_{k^{\prime}+1}^{\prime}}-\langle b\rangle_{G_{k^{\prime}}^{\prime}}=\left(\left|G_{k^{\prime}+1}^{\prime}\right|+\left|G_{k^{\prime}}^{\prime}\right|\right) \frac{F}{N}, \\
& \langle b\rangle_{G_{k, 2}^{\prime}}-\langle b\rangle_{G_{k, 1}^{\prime}} \leq \frac{F}{N}\left|G_{k}^{\prime}\right|, \quad \forall G_{k, 1}^{\prime}, G_{k, 2}^{\prime} \subsetneq G_{k}^{\prime}, \text { with } G_{k, 2}^{\prime}=G_{k}^{\prime} \backslash G_{k, 1}^{\prime}, \\
& \quad \forall k \in\{1, \ldots, M+1\} .
\end{aligned}
$$

For a cluster structure satisfying (4) the conditions (15) are satisfied. The lemma implies that if one of the inequalities of (15b) becomes an equality when $F$ is decreased continuously to $\tilde{F}$, then a cluster structure satisfying 
(17) can be identified by splitting one of the original clusters and leaving the other clusters unaltered.

If only one inequality of (15b) changes into an equality at $F=\tilde{F}$, then one can check that $(17 \mathrm{c})$ holds with strict inequality (see also the proof in the appendix the cases $k \in\left\{k^{\prime}, k^{\prime}+1\right\}$ ). Since (17a) and (17b) change into strict inequalities for $F$-values slightly less than $\tilde{F}$, the resulting cluster structure satisfies (4) for these values of $F$.

If more than one inequality changes into an equality at $F=\tilde{F}$, then (17c) still contains one or more equalities; the lemma can be applied several times until (17c) only consists of strict inequalities, again leading to a cluster structure that will satisfy (4) for values of $F$ slightly lower than $\tilde{F}$.

Proof of theorem 3. Pick an arbitrary $b$ and let $F$ be large enough, such that (4) holds for $G=(\{1, \ldots, N\})$, i.e. there is only one cluster. Let $F$ decrease and let $F^{\prime}$ denote the (largest) value of $F$ for which one of the inequalities of $(4 \mathrm{~b})$ becomes an equality. Application of the previous lemma (one or more times) leads to a new ordered set partition $G^{\prime}$ satisfying (4) for $F \in\left[F^{\prime \prime}, F^{\prime}\right)$, for some $F^{\prime \prime}<F^{\prime}$. This argument can be repeated to obtain a partition of the positive real axis in intervals for $F$; each interval corresponds to a cluster structure satisfying (4).

We have therefore shown that, given $b$ and $F$ we can construct an ordered set partition $G$ satisfying (4). Since (4) implies clustering behavior characterized by $G$, irrespective of the initial condition, and since a solution of (1) cannot exhibit clustering behavior with respect to different cluster structures, there exists a unique ordered set partition $G$ for every $b$ and $F \geq 0$, such that (4) holds.

Remark 5 From the proof of theorem 3 it follows that, when decreasing $F$ from a sufficiently large value, a tree structure relating $F$ with asymptotic cluster velocities can be identified; see Fig. 1(d) for a system of 7 agents. In the generic case there will be $N-1$ bifurcation values for $F$, each corresponding to a separation of a cluster into two new clusters. The tree structure can also be interpreted as a dendrogram, indicating that this model may lead to a hierarchical procedure for data clustering (see e.g. [13, 31, 15] for more information on this subject).

\section{Behavior within the clusters}

We consider a solution of the system (1) exhibiting clustering behavior characterized by the clusters $G_{1}, \ldots, G_{M}$ and the time $T$. We assume that $F>0$. 
Fix $k$; for $t>T$, the interaction between agents in $G_{k}$ and agents in other clusters can be replaced by the corresponding saturation value $\pm F$. We will show that, under some mild assumptions, the distances between $x$-values in the cluster $G_{k}$ will approach constant values that are independent of the initial condition. Let $\sigma$ be a bijection from $I_{k} \triangleq\left\{1, \ldots,\left|G_{k}\right|\right\}$ to $G_{k}$ and set

$$
\begin{gathered}
x_{i}^{\prime} \triangleq x_{\sigma(i)}-\langle x\rangle_{G_{k}}, \\
b_{i}^{\prime} \triangleq b_{\sigma(i)}-\langle b\rangle_{G_{k}},
\end{gathered}
$$

for all $i$ in $I_{k}$. Then for $t>T$ we obtain

$$
\begin{aligned}
\dot{x}_{i}^{\prime}(t) & =b_{\sigma(i)}+\frac{1}{N} \sum_{j=1}^{N} f\left(x_{j}(t)-x_{\sigma(i)}(t)\right)-\langle b\rangle_{G_{k}}-\frac{F}{N}\left(\sum_{k^{\prime}>k}\left|G_{k^{\prime}}\right|-\sum_{k^{\prime}<k}\left|G_{k^{\prime}}\right|\right) \\
& =b_{i}^{\prime}+\frac{1}{N} \sum_{j \in I_{k}} f\left(x_{\sigma(j)}(t)-x_{\sigma(i)}(t)\right),
\end{aligned}
$$

for all $i$ in $I_{k}$, or

$$
\dot{x}_{i}^{\prime}(t)=b_{i}^{\prime}+\frac{1}{N} \sum_{j \in I_{k}} f\left(x_{j}^{\prime}(t)-x_{i}^{\prime}(t)\right), \quad \forall i \in I_{k},
$$

which describes the dynamics of the agents belonging to $G_{k}$. The definition of $x^{\prime}$ implies that (18) is to be considered on $L_{0} \triangleq\left\{x^{\prime} \in \mathbb{R}^{\left|G_{k}\right|}:\left\langle x^{\prime}\right\rangle_{I_{k}}=0\right\}$. Consider the function

$$
W_{k}: \mathbb{R}^{\left|G_{k}\right|} \rightarrow \mathbb{R}: x^{\prime} \mapsto W_{k}\left(x^{\prime}\right)=\sum_{i \in I_{k}}-b_{i}^{\prime} x_{i}^{\prime}+\frac{1}{2 N} \sum_{i, j \in I_{k}} \int_{0}^{x_{i}^{\prime}-x_{j}^{\prime}} f(\tau) \mathrm{d} \tau .
$$

Then, $\forall i \in I_{k}$,

$$
\frac{\partial W_{k}}{\partial x_{i}^{\prime}}\left(x^{\prime}(t)\right)=-\dot{x}_{i}^{\prime}(t)
$$

and $W_{k}$ is a gradient function for the system (18). Since each $x_{i}^{\prime}(t)$ is bounded because of the clustering behavior, it follows that $x^{\prime}(t)$ will approach the set containing all equilibrium points of (18), which are the solutions of

$$
\begin{aligned}
& b_{i}^{\prime}+\frac{1}{N} \sum_{j \in I_{k}} f\left(x_{j}^{\prime}-x_{i}^{\prime}\right)=0, \quad \forall i \in I_{k}, \\
& \left\langle x^{\prime}\right\rangle_{I_{k}}=0 .
\end{aligned}
$$

We will prove that, under a mild assumption, (19) has only one solution. Assume that $x^{\prime e, 1}$ and $x^{\prime e, 2}$ are two solutions of (19), thus corresponding to two equilibrium solutions of (18). Consider the function

$$
V_{k}: \mathbb{R}^{\left|G_{k}\right|} \times \mathbb{R}^{\left|G_{k}\right|}:\left(x^{\prime 1}, x^{\prime 2}\right) \mapsto V_{k}\left(x^{\prime 1}, x^{\prime 2}\right) \triangleq \frac{1}{2} \sum_{i \in I_{k}}\left(x_{i}^{\prime 1}-x_{i}^{\prime 2}\right)^{2}
$$


For any two solutions $x^{\prime 1}$ and $x^{\prime 2}$ of the system (18) we can write

$$
\begin{aligned}
\frac{\mathrm{d}}{\mathrm{d} t}\left(V_{k}\left(x^{\prime 1}(t), x^{\prime 2}(t)\right)\right)=- & \frac{1}{N} \sum_{i \in I_{k}} \sum_{j \in I_{k}}\left(f\left(x_{j}^{\prime 1}(t)-x_{i}^{\prime 1}(t)\right)-f\left(x_{j}^{\prime 2}(t)-x_{i}^{\prime 2}(t)\right)\right) \\
& \times\left(\left(x_{j}^{\prime 1}(t)-x_{i}^{\prime 1}(t)\right)-\left(x_{j}^{\prime 2}(t)-x_{i}^{\prime 2}(t)\right)\right),
\end{aligned}
$$

for all $t$ in $\mathbb{R}$, in a similar way as the expression for $\dot{V}$ was derived in section 7.2. For $x^{\prime 1}$ and $x^{\prime 2}$ equal to the equilibrium solutions $x^{\prime e, 1}$ and $x^{\prime e, 2}$, this expression must be zero. Since each separate term is non-negative ( $f$ is non-decreasing), it follows that

$$
\left(f\left(x_{j}^{\mathrm{e}, 1}-x_{i}^{\mathrm{e}, 1}\right)-f\left(x_{j}^{\mathrm{e}, 2}-x_{i}^{\mathrm{e}, 2}\right)\right)\left(\left(x_{j}^{\mathrm{e}, 1}-x_{i}^{\prime \mathrm{e}, 1}\right)-\left(x_{j}^{\prime \mathrm{e}, 2}-x_{i}^{\prime \mathrm{e}, 2}\right)\right)=0, \quad \forall i, j \in I_{k},
$$

and thus

$$
f\left(x_{j}^{\mathrm{e}, 1}-x_{i}^{\prime \mathrm{e}, 1}\right)=f\left(x_{j}^{\mathrm{e}, 2}-x_{i}^{\mathrm{e}, 2}\right), \quad \forall i, j \in I_{k} .
$$

For the remainder of this section we assume that the inequalities in (4b) corresponding to $G_{k}$ are strict:

$$
\langle b\rangle_{G_{k, 2}}-\langle b\rangle_{G_{k, 1}}<\frac{F}{N}\left|G_{k}\right|, \quad \forall G_{k, 1}, G_{k, 2} \subsetneq G_{k}, \text { with } G_{k, 2}=G_{k} \backslash G_{k, 1},
$$

and that $f$ is strictly increasing in the interval $(-d, d)$. We will show that under these conditions the solution to (19) is unique; this is accomplished by showing that (20), together with (19b) considered for $x^{\prime e, 1}$ and $x^{\prime e, 2}$, implies that $x^{\mathrm{e}, 2}=x^{\mathrm{e}, 1}$. Since $W_{k}$ is a gradient function for (18), it follows that $x^{\mathrm{ee}, 1}$ is the only point of $L_{0}$ where the gradient of $W_{k}$ equals zero. The convexity of $W_{k}$ ( $W_{k}$ is convex as it is a sum of convex functions) then implies that the restriction of $W_{k}$ to $L_{0}$ attains an absolute minimum in $x^{\mathrm{e}, 1}$, and therefore $x^{\prime e, 1}$ is an asymptotically stable equilibrium point of (18) with respect to the state space $L_{0}$.

First we will prove that the undirected graph $\mathcal{G}\left(x^{\prime e, 1}\right)$, defined by connecting $i$ and $j$ if and only if $\left|x_{i}^{\mathrm{e}, 1}-x_{j}^{\prime \mathrm{e}, 1}\right|<d$, is connected. If this were not the case then we would be able to find a nonempty set $G_{k, 2}^{\prime} \subset I_{k}$ with $G_{k, 1}^{\prime}=I_{k} \backslash G_{k, 2}^{\prime}$ also nonempty, and such that

$$
x_{i}^{\prime \mathrm{e}, 1}-x_{j}^{\mathrm{e}, 1} \geq d, \quad \forall(i, j) \in G_{k, 2}^{\prime} \times G_{k, 1}^{\prime},
$$

implying that the interactions between agents of $G_{k, 2}^{\prime}$ and agents of $G_{k, 1}^{\prime}$ would be saturated. Then from (19a) it would follow that (by averaging over $G_{k, 2}^{\prime}$ 
resp. $\left.G_{k, 1}^{\prime}\right)$

$$
\begin{aligned}
& \left\langle b^{\prime}\right\rangle_{G_{k, 2}^{\prime}}-\frac{F}{N}\left|G_{k, 1}^{\prime}\right|=0, \\
& \left\langle b^{\prime}\right\rangle_{G_{k, 1}^{\prime}}+\frac{F}{N}\left|G_{k, 2}^{\prime}\right|=0,
\end{aligned}
$$

implying that

$$
\left\langle b^{\prime}\right\rangle_{G_{k, 2}^{\prime}}-\left\langle b^{\prime}\right\rangle_{G_{k, 1}^{\prime}}=\left|G_{k}\right| \frac{F}{N}
$$

and since $\left\langle b^{\prime}\right\rangle_{G_{k, 2}^{\prime}}-\left\langle b^{\prime}\right\rangle_{G_{k, 1}^{\prime}}=\langle b\rangle_{G_{k, 2}}-\langle b\rangle_{G_{k, 1}}$, with $G_{k, 1}=\sigma\left(G_{k, 1}^{\prime}\right)$ and $G_{k, 2}=\sigma\left(G_{k, 2}^{\prime}\right)$, this contradicts (21). It follows that the graph $\mathcal{G}\left(x^{\mathrm{e}, 1}\right)$ must be connected.

Since $f$ is strictly increasing in $(-d, d)$, the equation $f\left(x_{j}^{\prime \mathrm{e}, 1}-x_{i}^{\mathrm{e}, 1}\right)=f\left(x_{j}^{\mathrm{e}, 2}-\right.$ $\left.x_{i}^{\prime e, 2}\right)$ implies $x_{j}^{\prime e, 2}-x_{i}^{\prime e, 2}=x_{j}^{\prime e, 1}-x_{i}^{\prime e, 1}$ if $x_{j}^{\prime e, 1}-x_{i}^{\prime e, 1} \in(-d, d)$. The connectedness of $\mathcal{G}\left(x^{\mathrm{e}, 1}\right)$ implies that for any $i$ and $j$ in $I_{k}(i \neq j)$ we can find agents $l_{0}=i, l_{1}, \ldots, l_{P-1}, l_{P}=j$ (where $P$ denotes the corresponding path length) such that subsequent agents $l_{m-1}$ and $l_{m}$ have an edge of $\mathcal{G}\left(x^{\prime e, 1}\right)$ in common and thus $x_{l_{m}}^{\prime e, 2}-x_{l_{m-1}}^{\text {e,2 }}=x_{l_{m}}^{\text {e,1 }}-x_{l_{m-1}}^{\prime \text { e,1 }}$. Consequently

$$
\begin{aligned}
x_{j}^{\mathrm{e}, 2}-x_{i}^{\mathrm{e}, 2} & =\sum_{m=1}^{P} x_{l_{m}}^{\mathrm{e}, 2}-x_{l_{m-1}}^{\mathrm{e}, 2} \\
& =\sum_{m=1}^{P} x_{l_{m}}^{\prime \mathrm{e}, 1}-x_{l_{m-1}}^{\mathrm{e}, 1} \\
& =x_{j}^{\mathrm{e}, 1}-x_{i}^{\mathrm{e}, 1} .
\end{aligned}
$$

This implies $x_{i}^{\prime \mathrm{e}, 2}-\left\langle x^{\prime \mathrm{e}, 2}\right\rangle_{I_{k}}=x_{i}^{\prime \mathrm{e}, 1}-\left\langle x^{\mathrm{e}, 1}\right\rangle_{I_{k}}, \forall i \in I_{k}$, and since $\left\langle x^{\mathrm{e}, 2}\right\rangle_{I_{k}}=$ $\left\langle x^{\prime e, 1}\right\rangle_{I_{k}}=0$, it follows that $x^{\prime e, 2}=x^{\prime e, 1}$.

Remark 6 Notice that from the connectedness of $\mathcal{G}\left(x^{\text {ee,1 }}\right)$ it follows that

$$
\left|x_{j}^{\prime \mathrm{e}, 1}-x_{i}^{\mathrm{e}, 1}\right|<\left(\left|G_{k}\right|-1\right) d, \quad \forall(i, j) \in I_{k}^{2},
$$

providing an upper bound for the extent (in differences of $x_{i}$-values) of the cluster $G_{k}$ for large values of $t$.

\section{Conclusion}

We have introduced a dynamical model of non-identical agents, which, due to mutual attraction, organize themselves into clusters. Each agent has a natural velocity, and is attracted to all other agents according to a saturating interaction function. 
For any choice of the parameters a cluster structure emerges, characterized by a set of inequalities in the model parameters: the offset in average natural velocity between different clusters should outweigh their overall mutual attraction, while within a cluster, any partition into two subsets should result in a difference in average natural velocity that does not exceed their mutual attraction. In the long run the agents behave linearly in time (up to a constant) with a common asymptotic velocity for agents belonging to the same cluster. Moreover, the cluster structure is independent of initial conditions.

For varying coupling strength different cluster structures are obtained, ranging from a single cluster containing all agents to (in the generic case) trivial clusters, each containing one agent. We refer to some values of the coupling strength as bifurcation values: decreasing the coupling strength below such a transition value results in a new cluster structure which is a refinement of the previous cluster structure, i.e. generically the transition corresponds to splitting one cluster into two clusters.

The clustering behavior of our model, and the variation of the cluster structure with varying coupling strength is similar to the behavior of the Kuramoto model, while admitting a full mathematical analysis.

Furthermore the system equations naturally arise in diffusion/transportation problems, as has been illustrated by deriving the model equations from the physical laws governing a network of interconnected water basins.

\section{Acknowledgments}

We thank the referees for their constructive comments.

This paper presents research results of the Belgian Programme on Interuniversity Attraction Poles, initiated by the Belgian Federal Science Policy Office. The scientific responsibility rests with its authors.

During this research Filip De Smet was supported by a Ph.D. fellowship of the Research Foundation - Flanders (FWO).

\section{A Proof of lemma 4}

The inequalities (17a) are identical to inequalities from (15a), except for $k \in$ $\left\{k^{\prime}-1, k^{\prime}, k^{\prime}+1\right\}$. Equation (17b) is identical to (16) (and also implies (17a) for $\left.k=k^{\prime}\right)$. The inequalities (17c) are identical to inequalities from (15b), except 
for $k \in\left\{k^{\prime}, k^{\prime}+1\right\}$. Therefore, we only need to prove (17a) for $k \in\left\{k^{\prime}-1, k^{\prime}+1\right\}$ and (17c) for $k \in\left\{k^{\prime}, k^{\prime}+1\right\}$. The proofs consist of writing the left hand sides of (17) as linear combinations of the left hand sides of (15).

From

$$
\begin{gathered}
\langle b\rangle_{G_{k^{\prime}, 2}}-\langle b\rangle_{G_{k^{\prime}, 1}}=\frac{F}{N}\left|G_{k^{\prime}}\right| \\
\langle b\rangle_{G_{k^{\prime}}}=\frac{\left|G_{k^{\prime}, 2}\right|\langle b\rangle_{G_{k^{\prime}, 2}}+\left|G_{k^{\prime}, 1}\right|\langle b\rangle_{G_{k^{\prime}, 1}}}{\left|G_{k^{\prime}}\right|}
\end{gathered}
$$

it follows that

$$
\begin{aligned}
\langle b\rangle_{G_{k^{\prime}, 2}} & =\langle b\rangle_{G_{k^{\prime}}}+\frac{F}{N}\left|G_{k^{\prime}, 1}\right|, \\
\langle b\rangle_{G_{k^{\prime}, 1}} & =\langle b\rangle_{G_{k^{\prime}}}-\frac{F}{N}\left|G_{k^{\prime}, 2}\right|,
\end{aligned}
$$

and thus

$$
\begin{aligned}
\langle b\rangle_{G_{k^{\prime}+2}^{\prime}}-\langle b\rangle_{G_{k^{\prime}+1}^{\prime}} & =\langle b\rangle_{G_{k^{\prime}+1}}-\langle b\rangle_{G_{k^{\prime}, 2}} \\
& =\langle b\rangle_{G_{k^{\prime}+1}}-\langle b\rangle_{G_{k^{\prime}}}-\left|G_{k^{\prime}, 1}\right| \frac{F}{N} \\
& \geq\left(\left|G_{k^{\prime}+1}\right|+\left|G_{k^{\prime}}\right|\right) \frac{F}{N}-\left|G_{k^{\prime}, 1}\right| \frac{F}{N}, \quad \text { because of }(15 \mathrm{a}), \\
& =\left(\left|G_{k^{\prime}+1}\right|+\left|G_{k^{\prime}, 2}\right|\right) \frac{F}{N} \\
& =\left(\left|G_{k^{\prime}+2}^{\prime}\right|+\left|G_{k^{\prime}+1}^{\prime}\right|\right) \frac{F}{N}
\end{aligned}
$$

and

$$
\begin{aligned}
\langle b\rangle_{G_{k^{\prime}}^{\prime}}-\langle b\rangle_{G_{k^{\prime}-1}^{\prime}} & =\langle b\rangle_{G_{k^{\prime}, 1}}-\langle b\rangle_{G_{k^{\prime}-1}} \\
& =\langle b\rangle_{G_{k^{\prime}}}-\langle b\rangle_{G_{k^{\prime}-1}}-\left|G_{k^{\prime}, 2}\right| \frac{F}{N} \\
& \geq\left(\left|G_{k^{\prime}}\right|+\left|G_{k^{\prime}-1}\right|\right) \frac{F}{N}-\left|G_{k^{\prime}, 2}\right| \frac{F}{N}, \quad \text { because of (15a), } \\
& =\left(\left|G_{k^{\prime}-1}\right|+\left|G_{k^{\prime}}\right|-\left|G_{k^{\prime}, 2}\right|\right) \frac{F}{N} \\
& =\left(\left|G_{k^{\prime}-1}^{\prime}\right|+\left|G_{k^{\prime}}^{\prime}\right|\right) \frac{F}{N}
\end{aligned}
$$

proving (17a).

Regarding (17c) for $k=k^{\prime}+1$, choose an arbitrary nonempty subset $G_{k^{\prime}+1,2}^{\prime} \subsetneq$ 
$G_{k^{\prime}+1}^{\prime}=G_{k^{\prime}, 2}$ and set $G_{k^{\prime}+1,1}^{\prime}=G_{k^{\prime}+1}^{\prime} \backslash G_{k^{\prime}+1,2}^{\prime}$. From

$$
\begin{gathered}
\langle b\rangle_{G_{k^{\prime}+1}^{\prime}}-\langle b\rangle_{G_{k^{\prime}}^{\prime}}=\frac{\left|G_{k^{\prime}+1,1}^{\prime}\right|\langle b\rangle_{G_{k^{\prime}+1,1}^{\prime}}+\left|G_{k^{\prime}+1,2}^{\prime}\right|\langle b\rangle_{G_{k^{\prime}+1,2}^{\prime}}}{\left|G_{k^{\prime}+1}^{\prime}\right|}-\langle b\rangle_{G_{k^{\prime}}^{\prime}}, \\
\langle b\rangle_{G_{k^{\prime}+1,2}^{\prime}}-\langle b\rangle_{G_{k^{\prime}}^{\prime} \cup G_{k^{\prime}+1,1}^{\prime}}=\langle b\rangle_{G_{k^{\prime}+1,2}^{\prime}}-\frac{\left|G_{k^{\prime}}^{\prime}\right|\langle b\rangle_{G_{k^{\prime}}^{\prime}}+\left|G_{k^{\prime}+1,1}^{\prime}\right|\langle b\rangle_{G_{k^{\prime}+1,1}^{\prime}}}{\left|G_{k^{\prime}}^{\prime}\right|+\left|G_{k^{\prime}+1,1}^{\prime}\right|}
\end{gathered}
$$

one can easily verify that

$$
\begin{aligned}
& \langle b\rangle_{G_{k^{\prime}+1,2}^{\prime}}-\langle b\rangle_{G_{k^{\prime}+1,1}^{\prime}}=\frac{\left|G_{k^{\prime}+1}^{\prime}\right|}{\left(\left|G_{k^{\prime}}^{\prime}\right|+\left|G_{k^{\prime}+1}^{\prime}\right|\right)\left|G_{k^{\prime}+1,1}^{\prime}\right|} \\
& \times\left(\left(\left|G_{k^{\prime}}^{\prime}\right|+\left|G_{k^{\prime}+1,1}^{\prime}\right|\right)\left(\langle b\rangle_{G_{k^{\prime}+1,2}^{\prime}}-\langle b\rangle_{G_{k^{\prime}}^{\prime} \cup G_{k^{\prime}+1,1}^{\prime}}^{\prime}\right)-\left|G_{k^{\prime}}^{\prime}\right|\left(\langle b\rangle_{G_{k^{\prime}+1}^{\prime}}-\langle b\rangle_{G_{k^{\prime}}^{\prime}}\right)\right),
\end{aligned}
$$

which can be bounded by using (15b) with $k=k^{\prime}, G_{k, 2}=G_{k^{\prime}+1,2}^{\prime}, G_{k, 1}=$ $G_{k^{\prime}}^{\prime} \cup G_{k^{\prime}+1,1}^{\prime}$ and (17b) which is the same as (16):

$$
\begin{aligned}
\langle b\rangle_{G_{k^{\prime}+1,2}^{\prime}}-\langle b\rangle_{G_{k^{\prime}+1,1}^{\prime}} \leq & \frac{\left|G_{k^{\prime}+1}^{\prime}\right|}{\left(\left|G_{k^{\prime}}^{\prime}\right|+\left|G_{k^{\prime}+1}^{\prime}\right|\right)\left|G_{k^{\prime}+1,1}^{\prime}\right|} \\
& \quad \times\left(\left(\left|G_{k^{\prime}}^{\prime}\right|+\left|G_{k^{\prime}+1,1}^{\prime}\right|\right)-\left|G_{k^{\prime}}^{\prime}\right|\right) \frac{F}{N}\left(\left|G_{k^{\prime}}^{\prime}\right|+\left|G_{k^{\prime}+1}^{\prime}\right|\right) \\
= & \frac{F}{N}\left|G_{k^{\prime}+1}^{\prime}\right| .
\end{aligned}
$$

The inequalities (17c) for $k=k^{\prime}$ are proven analogously.

\section{References}

[1] D. Aeyels and F. De Smet. A model for the dynamical process of cluster formation. In 7th IFAC Symposium on Nonlinear Control Systems (NOLCOS 2007), pages 260-264, August 2007.

[2] D. Aeyels and J. A. Rogge. Existence of partial entrainment and stability of phase locking behavior of coupled oscillators. Progress of Theoretical Physics, 112(6):921-942, December 2004.

[3] C. M. Bordogna and E. V. Albano. Statistical methods applied to the study of opinion formation models: a brief overview and results of a numerical study of a model based on the social impact theory. Journal of Physics: Condensed Matter, 19(6):065144 (16pp), February 2007.

[4] A. Czirók, E. Ben-Jacob, I. Cohen, and T. Vicsek. Formation of complex bacterial colonies via self-generated vortices. Physical Review E, 54(2):1791-1801, August 1996. 
[5] H. Daido. Multibranch entrainment and scaling in large populations of coupled oscillators. Physical Review Letters, 77(7):1406-1409, August 1996.

[6] F. De Smet and D. Aeyels. Partial entrainment in the finite KuramotoSakaguchi model. Physica D, 234(2):81-89, October 2007.

[7] F. De Smet and D. Aeyels. Clustering in a network of non-identical and mutually interacting agents. Submitted to Proceedings of the Royal Society A, 2008.

[8] F. De Smet and D. Aeyels. Resonances and entrainment break up in Kuramoto models with multimodal frequency densities. Accepted for publication in Physical Review E, 2008.

[9] G. Deffuant, F. Amblard, G. Weisbuch, and T. Faure. How can extremism prevail? a study based on the relative agreement interaction model. Journal of Artificial Societies and Social Simulation, 5(4), 2002.

[10] D. Golomb, D. Hansel, B. Shraiman, and H. Sompolinsky. Clustering in globally coupled phase oscillators. Physical Review A, 45(6):3516-3530, March 1992.

[11] G. Grégoire, H. Chaté, and Y. Tu. Moving and staying together without a leader. Physica D, 181(3-4):157-170, July 2003.

[12] R. Hegselmann and U. Krause. Opinion dynamics and bounded confidence: models, analysis and simulation. Journal of Artificial Societies and Social Simulation, 5(3), 2002.

[13] A. K. Jain, M. N. Murty, and P. J. Flynn. Data clustering: A review. ACM Computing Surveys, 31(3):264-323, September 1999.

[14] Y. Kuramoto. Cooperative dynamics of oscillator community. Supplement of the Progress of Theoretical Physics, 79:223-240, 1984.

[15] T. W. Liao. Clustering of time series data-a survey. Pattern Recognition, 38(11):1857-1874, November 2005.

[16] W. Lohmiller and J. Slotine. On contraction analysis for non-linear systems. Automatica, 34(6):683-696, 1998.

[17] Y. Maistrenko, O. Popovych, O. Burylko, and P. A. Tass. Mechanism of desynchronization in the finite-dimensional Kuramoto model. Physical Review Letters, 93(8):084102, August 2004.

[18] S. C. Manrubia, A. S. Mikhailov, and D. H. Zanette. Emergence of Dynamical Order: Synchronization Phenomena in Complex Systems, volume 2 of World Scientific Lecture Notes in Complex Systems. World Scientific, 2004.

[19] A. S. Mikhailov and D. H. Zanette. Noise-induced breakdown of coherent collective motion in swarms. Physical Review E, 60(4):4571+, October 1999.

[20] L. G. Morelli, H. A. Cerdeira, and D. H. Zanette. Frequency clustering of coupled phase oscillators on small-world networks. European Physical Journal B, 43:243-250, Jan. 2005.

[21] K. Okuda. Variety and generality of clustering in globally coupled oscillators. Physica D, 63(3-4):424-436, 1993. 
[22] A. Pavlov, A. Pogromsky, N. van de Wouw, and H. Nijmeijer. Convergent dynamics, a tribute to Boris Pavlovich Demidovich. Systems \& Control Letters, 52:257-261, July 2004.

[23] J. E. Plapp. Engineering Fluid Mechanics. Series in Engineering of the Physical Sciences. Prentice Hall, 1968.

[24] C. W. Reynolds. Flocks, herds, and schools: A distributed behavioral model. Computer Graphics, 21(4):25-34, 1987. (SIGGRAPH '87 Conference Proceedings).

[25] S. H. Strogatz. From Kuramoto to Crawford: exploring the onset of synchronization in populations of coupled oscillators. Physica D, 143:120, 2000.

[26] S. H. Strogatz. SYNC: The Emerging Science of Spontaneous Order. Hyperion Press, New York, 2003.

[27] K. Sznajd-Weron and J. Sznajd. Opinion evolution in closed community. International Journal of Modern Physics C, 11(6):1157-1165, September 2000.

[28] P. Tass. Phase and frequency shifts in a population of phase oscillators. Physical Review E, 56(2):2043-2060, August 1997.

[29] F. Wu and B. A. Huberman. Social structure and opinion formation. Computational Economics 0407002, EconWPA, July 2004.

[30] D. H. Zanette and A. S. Mikhailov. Dynamical clustering in oscillator ensembles with time-dependent interactions. Europhysics Letters, 65(4):465-471, February 2004.

[31] Y. Zhao and G. Karypis. Data clustering in life sciences. Molecular Biotechnology, 31(1):55-80, September 2005. 